\title{
Energy Modeling with Nonlinear-Autoregressive Exogenous Neural Network
}

\author{
Syed Asad Hussain ${ }^{1 *}$, Richard Kwok Kit Yuen², Eric Wai Ming Lee \\ Department of Architecture and Civil Engineering, City University of Hong Kong, Kowloon, Hong Kong
}

\begin{abstract}
The model-based predictive control (MPC) is considered to be an effective tool for optimal control of building heating, ventilation, and air-conditioning (HVAC) systems. MPC need to update the operating set points of the local control loops that have a significant influence on the energy performance of the system. Performance of MPC relies on the accuracy of the system performance model. There are two commonly used modeling approach - conventional or analytical approach that is the way of process modeling for some time, but it tends to increase the online computational load as it requires a full mathematical description of the real system. Furthermore, such techniques rely on different simplifying assumptions that limit the accuracy of the performance model. A second commonly used technique is the data-driven approach. The neural network $(\mathrm{NN})$ is the most potent data-driven approach. $\mathrm{NN}$ can accurately model complex nonlinear systems without even knowing the structure of the system and it also addresses the problem of the online computational load since the computational load moves to the offline training step.
\end{abstract}

In order to set up neural network model-based predictive control (NNMPC), it is important to build a reliable energy model of HVAC system that can be used to perform multi-step-ahead prediction of system energy performance. In this paper, the energy modeling of the chiller plant is conducted. Data for the training of chiller plant energy model is generated from HVAC testbed build in TRNSYS simulation environment. The nonlinear-autoregressive neural network with exogenous input (NARX) is used to model the energy performance of the chiller plant. The NARX is a powerful method for forecasting of time series data and dynamic control problems. NARX model is first trained in the open-loop form with the actual output instead of feedback, using back-propagation with the Levenberg-Marquardt method; this model can be used to perform only one-step-ahead prediction. Open-loop NARX model is transformed into a closedloop form, by connecting the internal feedback, i.e. actual output is replaced by predicted output, to perform multi-step-ahead prediction (for predictive control).

Comparative analysis of developed NARX-based chiller model is carried out with respect to process data from testbed, which demonstrated the good accuracy of the NARX-based chiller model.

Keywords: model-based predictive control, heating, ventilation and air-conditioning, neural network, nonlinear-autoregressive model with exogenous input

\section{Introduction}

Building sector consumes about $40 \%$ of total energy and contributes about $33 \%$ of $\mathrm{CO}_{2}$ emissions in the world. Heating ventilation and air-conditioning (HVAC) systems are responsible for about $50 \%$ of the energy consumed by the building [1]. It has been welldemonstrated that deficient HVAC operation and control result in poor energy performance. Therefore, it is imperative to investigate potential methods to conserve energy usage of these systems.

HVAC systems are one of the most complex and challenging systems to control due to subsystem interaction, nonlinear dynamics, constrained operations and uncertainties due to the inaccuracy of sensors and variable load demand [2-5].

Over the decades, the traditional Proportional-IntegralDerivative (PID) control has been popular due to its

\footnotetext{
* Corresponding author: sahussain2@cityu.edu.hk
} 
simplicity, flexibility, ability to deliver adequate performance for most control problems, etc. However, PID has been well-known for its inability in handling processes with nonlinear dynamics, processes with delay and processes with signal noise. Model predictive controller (MPC) has been known as receding horizon control [6], after PID could be considered a strong candidate for HVAC control because of slow-moving dynamic control and it possesses the capability to handle uncertainties and constraints in a systematic manner. MPC integrates system model for predictive control rather than corrective control. It is well suited to slowmoving systems; use of cost function and optimization algorithm for optimal control, integration of energy conservation scheme in controller synthesis; and has the ability to cope up with a wide range of operation conditions $[7,8]$.

Performance of MPC is highly dependent on the accuracy system model. Therefore, synthesis of an accurate system model is essential for achieving reliability, optimal operation of HVAC systems. There are two commonly used modeling schemes (i) conventional or analytical approach and (ii) data-driven or statistical approach. These models rely on the physical laws and principles to provide fundamental knowledge of the system. In the past, a large number of models has been documented. The reference manual HVACSIM + building system and equipment's enlisted 26 dynamic models for HVAC components [9]. A thorough review of dynamic models for HVAC systems is presented in ASHRAE reference guide [10]. These models rely on different simplifying assumptions that limit the potency of synthesizing an accurate process model. Secondly, since these models rely on detail mathematical description of the system, therefore, they tend to increase the online computational load. It is very important to find the right balance between reliable process model and computational loading [11]. Computational loading increases (for MPC optimization problem) as the first principle model becomes more and more complicated.

A second commonly used modeling scheme is data driven or statistical approach. Artificial neural network (ANN) is the most potent data-driven approach. Without even knowing the structure of the system, ANN can model complex nonlinear systems which make it a promising alternative for conventional approaches. ANN addresses the problem of computational loading as it requires comparatively less computational effort. It was also suggested by Afram A. et. al. [12] to investigate nonlinear modeling schemes such as ANN with MPC. The nonlinear-autoregressive neural network with exogenous input (NARX) is a powerful method for modeling time series data $[13,14]$, can be used to model the HVAC systems energy performance. This paper presents the energy modeling of the chiller plant with the NARX model.

This study is organized as follows: Section 2 presents the identification of chiller plant energy model using the NARX model. Section 3 presents the performance of developed NARX energy model with respect to process data from the test bed. The conclusion is presented in Section 4.

\section{Identification using NARX model}

The nonlinear autoregressive network with exogenous inputs (NARX) is a recurrent dynamic network, useful for modeling time-series data. It is based on the linear autoregressive exogenous model. The mathematical description of NARX model is

$$
y(k)=f\left(\begin{array}{l}
y(k-1), y(k-2), \ldots, y\left(k-n_{y}\right), \\
u(k-1), u(k-2), \ldots, u\left(k-n_{u}\right)
\end{array}\right)
$$

where $y(k)$ and $u(k)$ are model output and input respectively, $n_{y}$ and $n_{u}$ are the respective leg space, $k$ represents the discrete time step, $f$ represents the nonlinear correlation between $y(k)$ and $u(k)$. Here the present value of $y(k)$ depends on the past values of $y(k)$ and $u(k)$.

In this work, the multilayer perceptron network (MLP) is selected as internal architecture for the NARX model. MLP network consists of several layers of interconnected neurons in a feedforward manner. In this study, the network architecture consists of one input layer, one output layer and one hidden layer as shown in Figure 1.

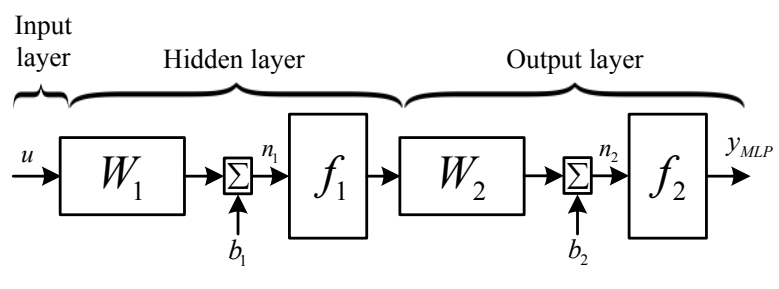

Figure 1. Multilayer perceptron network.

The output from MLP can be given by

$$
\begin{gathered}
n_{1}=b_{1}+W_{1}(u) \\
n_{2}=b_{1}+W_{2}\left(f_{1}\left(n_{1}\right)\right) \\
y_{M L P}=f_{2}\left(n_{2}\right)
\end{gathered}
$$

where $u$ represents input values, $y_{M L P}$ represents output values, $W$ represents synaptic weights connecting layers, $b_{1}$ and $b_{2}$ represent bias added to the hidden layer and output layer respectively and $f_{1}$ and $f_{2}$ represent the activation function for hidden layer and output layer respectively. In this study, hyperbolic tangent sigmoid (tansig) function [15] is used as nonlinear activation function

$$
f_{n, \text { tansig }}\left(n_{n}\right)=\frac{2}{1+e^{-2 n}}-1
$$

The training of feedforward network is done in openloop form as shown in Figure 2, since the actual past output is available while training. The Eq (1) is implemented as delay line (DL) to provide the memory element to the network and ability to capture the dynamics of the nonlinear system. The back-propagation with the Levenberg-Marquardt method is used to train the network since it has proven to be more efficient as compared to gradient-based methods [16]. 


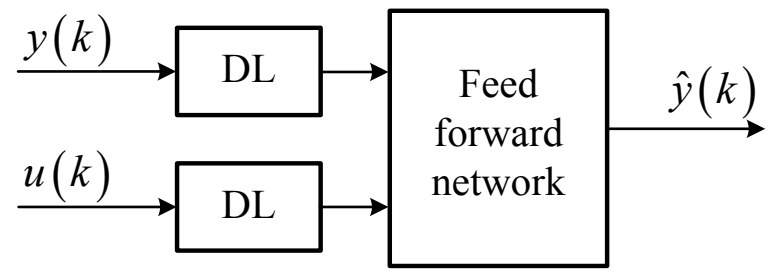

Figure 2. Open-loop form.

This open-loop NARX model can only provide a onestep-ahead prediction. Since multi-step-ahead prediction is required for the implementation of neural network model-based predictive control. The open-loop NARX model is transformed into closed-loop form, by connecting the internal feedback, i.e. actual past output is replaced by past predicted output from the model, to perform multi-step-ahead prediction.

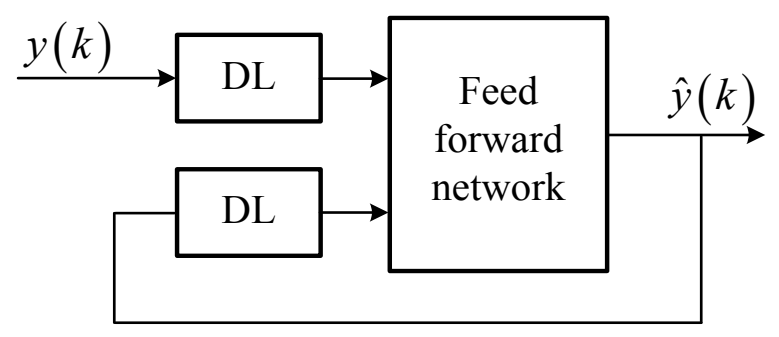

Figure 3. Closed-loop form.

\section{Case study}

A dynamic simulation testbed of the HVAC system was constructed in the TRNSYS simulation environment. The HVAC system used for this study consists of one condenser water loop, one chilled water loop, and one air loop. Data from this testbed was used for the training of NARX model within open loop form. The condenser water return temperature, chilled water supply temperature, and cooling load demand were presented as input for the training, while chiller power consumption was used as a target output. The network considered here had three layers: one input layer, one output layer, and one hidden layer. The hidden layer consists of five neurons and the output layer consists of one neuron. Mean-square-error (MSE) was considered as a cost function in the training process. The training was done with $80 \%$ of the data, while $20 \%$ of the data was used for validation to avoid overfitting. The trained NARX model was tested in the closed-loop form to judge the multistep-ahead prediction performance. Comparison of prediction accuracy between proposed NARX model and conventional physical model [17] was carried out based on mean square error.

\subsection{Performance analysis}

A comparison between the actual power consumption of the chiller plant and the predicted power consumption from the chiller physical model is presented in Figure $4 \mathrm{a}$, where, red lines indicate $95 \%$ performance bounds. This model provides reasonable performance. However, there is a slight model mismatch during high load period. On the other hand, NARX-based chiller model provided improved performance. Figure $4 \mathrm{~b}$, present the prediction performance of the NARX model. A good agreement can be observed between the actual and predicted operational data points, over the entire range.

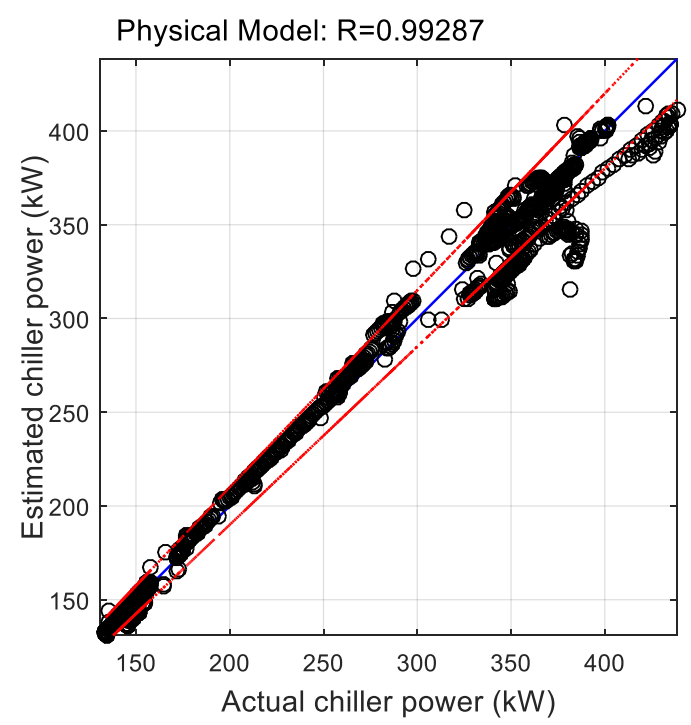

(a)

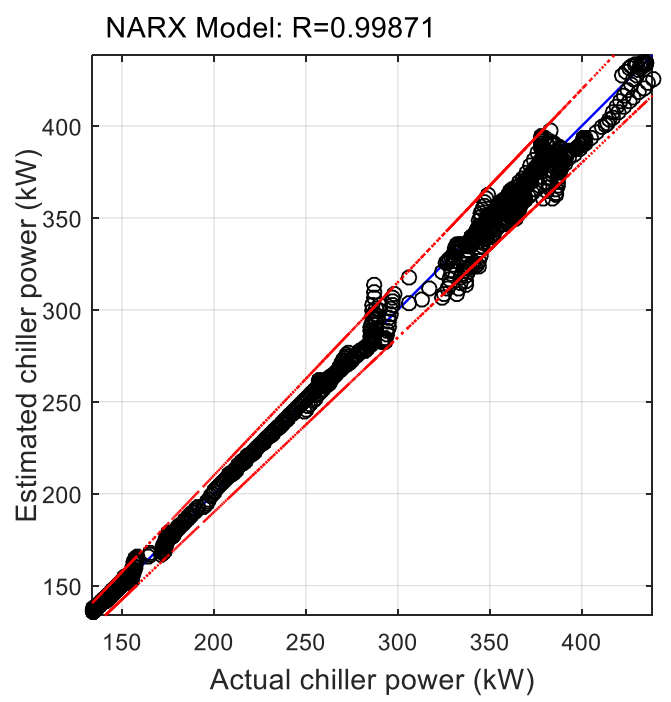

(b)

Figure 4. Prediction performance.

The MSE between the actual power and predicted chiller power consumption was 174.7254 and 24.7576 for physical and NARX model, respectively. Thus, the proposed NARX model demonstrates about 85\% improvement in accuracy as compared to the physical model. The error distribution for both models is presented in Figure 5, which shows that the NARX model produces error centered at zero, while a bias is evident for the physical model. 


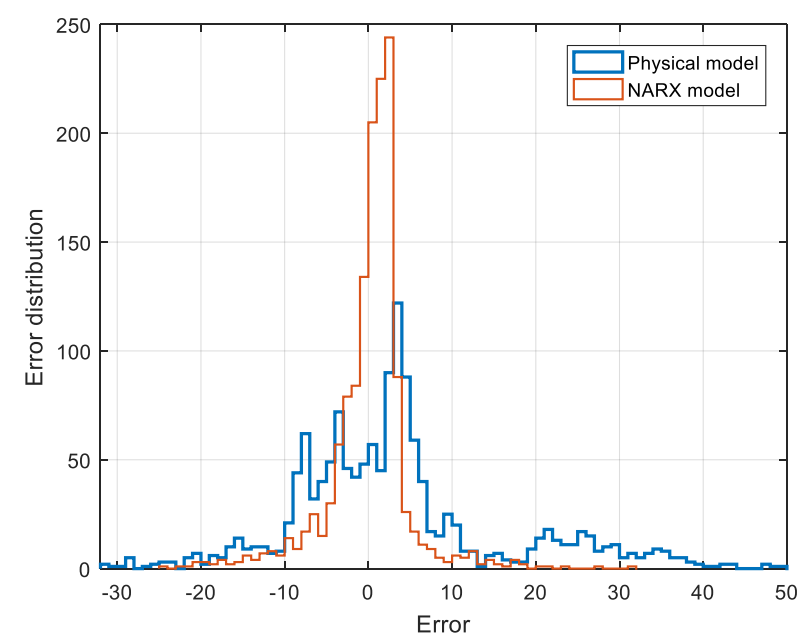

Figure 5. Error distribution.

\section{Conclusion}

This study presented a new dynamic model for chiller plant i.e. is capable of performing multi-step-ahead prediction. The nonlinear-autoregressive neural network with exogenous input (NARX) model was utilized in this study. The NARX model was trained in open-loop form and then transformed to closed-loop form to perform multi-step-ahead prediction. The case study was carried out to analyze the performance of NARX-based chiller model. Overall, the proposed NARX model was able to provide $85 \%$ improvement in accuracy as compared to the conventional physical model. In the future work, energy modeling of other subsystems of the HVAC system will be carried out using the same methodology and a supervisory model-based predictive control approach will be synthesized that will ensure energy efficient operation of HVAC systems.

\section{Acknowledgment}

The work described in this paper was supported by grants from the Research Grants Council of the Hong Kong Special Administrative Region, China (Project No. 11209518).

\section{References}

1. Pérez-Lombard, L., Ortiz, J. and Pout, C., 2008. A review on buildings energy consumption information. Energy and buildings, 40(3), pp.394398.

2. Semsar-Kazerooni, E.; Yazdanpanah, M.J.; Lucas, C. (2008) Nonlinear Control and Disturbance Decoupling of HVAC Systems Using Feedback Linearization and Backstepping With Load Estimation, in Control Systems Technology, IEEE Transactions, 16(5), 918-929.

3. Singhal A., Salsbury T. I. (2007) Characterization and cancellation of static nonlinearity in HVAC systems, ASHRAE Transactions, 113(1).
4. Huang G. Wang S., Xu X. (2009) Robust Model Predictive Control of VAV Air-Handling Units Concerning Uncertainties and Constraints, HVAC\&R Research, 16(1), 15-33.

5. Liao, Y., Sun, Y. and Huang, G., 2015. Robustness analysis of chiller sequencing control. Energy Conversion and Management, 103, pp.180-190.

6. Maciejowski J. M. (2002) Predictive control with constraints. Practice hall.

7. Huang G. (2011) Model predictive control of VAV zone thermal systems concerning bi-linearity and gain nonlinearity, Control Engineering Practice 19, $700-710$.

8. Huang G. Wang S.W., Xu X.H., (2009) A robust MPC strategy for improving control performance of air conditioning systems, Energy Conversion and Management 50, 2650-2658.

9. Clark D.R., in U.S.D. Commerce, N.B.o. Standarts, N.E.o. Laboratory, C. Technology, B.E,f.B, Division (Eds.), (1985) HVACSIM+ Building Systems and Equipment Simulation Program Reference Manual. Gaithersburg, MD 20899.

10. Bourdouxhe J.P., Groodent M., LeBrun J., (1998) Reference Guide for Dynamic Models of HVAC Equipment: American Society of Heating, Refrigerating and Air Conditioning Engineers Incorporated.

11. Cristea, V.M., Letiţia, T.O.M.A. and AGACHI, P.Ş., 2007. Simulation and Model Predictive Control of the Fluid Catalytic Cracking Unit Using Artificial Neural Networks. Revue Roumaine de Chimie, 52(12), pp.1157-1166.

12. Afram, A. and Janabi-Sharifi, F., 2014. Theory and applications of HVAC control systems-A review of model predictive control (MPC). Building and Environment, 72, pp.343-355.

13. Pisoni, E.; Farina, M.; Carnevale, C.; Piroddi, L. Forecasting peak air pollution levels using NARX models. Eng. Appl. Artif. Intell. 2009, 22, 593-602.

14. Ruiz, L.G.B.; Cuéllar, M.P.; Calvo-Flores, M.D.; Jiménez, M.D.C.P. An Application of Non-Linear Autoregressive Neural Networks to Predict Energy Consumption in Public Buildings. Energies 2016, 9, 684.

15. Vogl, T.P., Mangis, J.K., Rigler, A.K., Zink, W.T. and Alkon, D.L., 1988. Accelerating the convergence of the back-propagation method. Biological cybernetics, 59(4-5), pp.257263.

16. Hagan, M.T. and Menhaj, M.B., 1994. Training feedforward networks with the Marquardt algorithm. IEEE transactions on Neural Networks, 5(6), pp.989-993.

17. Asad, H.S., Yuen, R.K.K. and Huang, G., 2017. Multiplexed real-time optimization of HVAC systems with enhanced control stability. Applied Energy, 187, pp.640-651. 